\title{
Performance Analysis of a Mesh Satellite System based on Linear and Continuous Phase Modulations
}

\author{
R. Baroni*, F. Lombardo*, R. Suffritti ${ }^{\dagger}$, E.A. Candreva*†, A. Vanelli-Coralli*, G.E. Corazza*, \\ G. Colavolpe ${ }^{\ddagger}$, G. Gallinaro ${ }^{\S}$, N. Alagha \\ *DEIS/ARCES - University of Bologna, Viale Risorgimento 2, 40136 Bologna, Italy \\ e-mail: \{flombardo, rbaroni, ecandreva, avanelli, gecorazza\}@arces.unibo.it \\ ${ }^{\dagger}$ MAVIGEX,Strada Maggiore 63, 40125 Bologna, Italy \\ e-mail: \{rsuffritti, eacandreva\} @ mavigex.com \\ ${ }_{\ddagger}^{\ddagger}$ DII - University of Parma, Viale G. P. Usberti 181/A, 43100 Parma, Italy \\ e-mail: giulio@unipr.it \\ $\S$ Space Engineering S.p.A., Via dei Berio 91, 00155 Rome, Italy \\ e-mail: gennaro.gallinaro@space.it
}

\begin{abstract}
IEuropean Space Research and Technology Centre, European Space Agency, 2201 AZ Noordwijk, The Netherlands e-mail: Nader.Alagha@esa.int
\end{abstract}

\begin{abstract}
This paper addresses detailed waveform trade-offs for mesh satellite networks. The waveform analysis is carried out considering spectral efficiency, resilience against non linear distortion, channel impairments and interference for both linear and continuous phase modulation schemes defined in the DVBRCS2 standard.
\end{abstract}

\section{INTRODUCTION}

In a mesh satellite architecture two satellite terminals communicate directly through the satellite without accessing the satellite gateway (Fig. 1), thus avoiding a double hop link, which is typical for star configurations ${ }^{1}$. Mesh satellite architectures are therefore characterized by a lower transmission latency that enables higher quality of service for delay sensitive applications, e.g., voice communications, teleconferencing, etc. In addition, when multibeam architectures are also considered, mesh configurations allow for a higher traffic flexibility thanks to the capability of the satellite payload to routing traffic to different beams. In this paper, we address a multibeam mesh architecture based on the new DVB-RCS2 [1] defined waveforms, i.e., linear modulation (LM) and continuous phase modulation (CPM), with the aim of evaluating waveform performance under the constraints introduced by the mesh architecture. In fact, the ultimate goal of this work is to give a detailed insight on the selected waveform behavior in

\footnotetext{
${ }^{1}$ Double hop link means that the signal from User A goes to User B through the gateway, i.e., through the following path: User A-satellitegateway-satellite-User B. Hence, the information transits through the satellite twice.
}

the described scenarios in order to provide to system designers and operators a tool for the optimal configuration selection.

Satellite mesh networks have been considered in [2], [3] for the case of single beam or for statically-connected multiple beams. The use of regenerative payload has been addressed in [4], while semi regenerative payloads have been considered in [5],[6],[7]. In [8] different architectures for mesh satellite networking have been designed providing link budgets and setting up the framework for a detailed analysis of the waveforms performance in mesh systems reported in this paper. More in detail, reference [8] provides the key elements of the mesh satellite network, which has been designed considering case specific optimizations as well as input from previous studies [2]-[7]. In this paper we move from the system view to the link view, focusing on link-level aspects and impairments.

The performance assessment we provide is carried out by taking into account channel imperfections, i.e., adjacent channel interference, phase noise, and frequency offset, as well as transmitter non-linearities. It is worthwhile noting that the latter aspect is magnified in mesh systems because at both ends of the communication link a small antenna is used. As a matter of fact, in a double hop link the antenna gateway, that acts as receiving antenna in the first hop and as transmitting antenna in the second hop, is typically characterized by an extremely large gain, that attenuates the link budget challenges. In a single hop, i.e., mesh link, the communication link terminates at both ends at the user terminal that is equipped with a small and low cost antenna. In addition, the need of keeping the terminal cost at a market competitive level imposes also 
the use of low cost amplifiers at the user side that further exacerbate the non-linear distortion effects.

In this framework, we evaluate the trade-off between error rate performance, resilience to receiver instabilities, robustness against non-linearities and achievable spectral efficiency. In fact, linear modulations are able to provide high spectral efficiencies, but at the cost of highly varying envelope that imposes stringent requirements in terms of amplifiers backoff. On the other hand, a Continuous Phase Modulation offers an extremely useful constant envelope, that allows the signal to pass undistorted through the power amplifier without any need of back-off, which directly translates in power savings, but at the expense of a limited spectral efficiency.

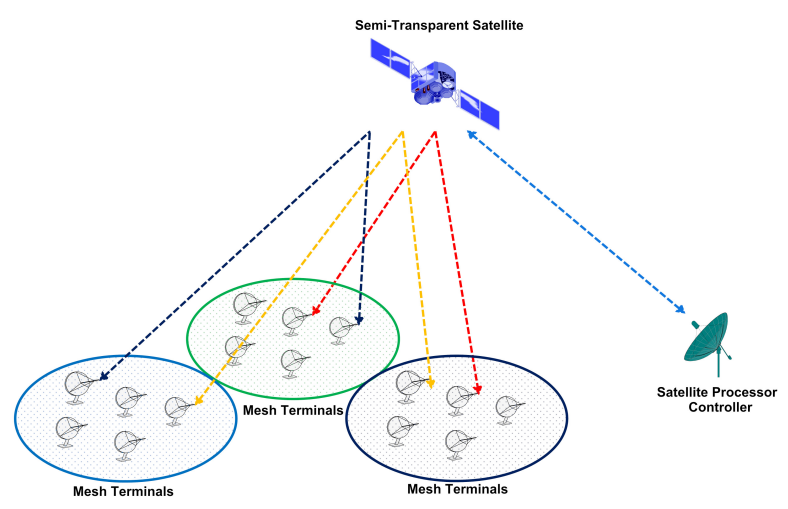

Fig. 1. Mesh System Scenario

\section{System MOdeL}

\section{A. Satellite mesh scenario}

The considered system is based on the mesh scenario already depicted in Fig. 1. A semi-regenerative satellite segment is considered [5]-[6]-[7], in order to achieve full-mesh connectivity of multiple terminals without the need of onground Hub stations.

In such scenario, while the satellite HPA is forced to work in its linear region to avoid non-linear distortion on the multiplex that would affect the frequency orthogonality, user terminals are characterized by low cost HPAs working as much as possible close to saturation. Hence, in-band distortion as well as out-of-band spectral regrowth are to be expected on the user side. Furthermore, because of the need of keeping the user terminal at a competitive cost, the received signal is likely to be strongly affected by phase noise and frequency errors.

Therefore, as indicated in the introduction, in this paper we consider LM and CPM waveforms as defined in the new DVB-RCS2 standard [1]. The selection of such standard has been based on the rationale that the waveforms have been designed for a mass-market terminal-to-satellite link very similar to those experienced in a single hop mesh network. In addition, the models for channel impairments we assume in this study are derived from the ones adopted in the DVB-RCS2 standardization process, i.e., the frequency error has uniform statistic over $[-4 k H z, 4 k H z]$, the phase noise model is that used in [1], and for adjacent channel interference (ACI) we assume a total of 6 balanced interferers with the same power of the useful signal.

\section{B. Linear Modulation Waveforms}

Linear modulation schemes adopted in the DVB-RCS2 standard are based on QPSK , 8PSK and 16QAM constellations and a 16 circular states turbocode (known also as Turbo- $\Phi$ ) with nominal code rate equal to $1 / 3$. Different code rates can be obtained through puncturing. The interleaver (embedded in the turbo encoder) and the bit order are specific for each modulation and code-rate combination. In total, there are 30 possible DVB-RCS2 LM waveforms.

In this work, two waveforms have been selected as benchmark, corresponding to QPSK with code rate $1 / 3$, and 8PSK with code rate $2 / 3$, and their additional parameters are summarized in Table I along with other system values and simulation parameters. The choice of the modulation parameters has been carried out with the aim of providing a fair comparison, in terms of spectral efficiency, between LM and CPM waveforms.

TABLE I

LiNEAR MODUlation WAVEFORM PARAMETERS

\begin{tabular}{|l|c|c|}
\hline DVB-RCS2 Waveform ID & 3 & 8 \\
\hline Modulation & QPSK & 8PSK \\
\hline Code-rate & $1 / 3$ & $2 / 3$ \\
\hline Packet length (bit) & 304 & 920 \\
\hline Roll-off & \multicolumn{2}{|c|}{0.2} \\
\hline Spectral efficiency & 0.606 & 1.818 \\
\hline HPA type & Ka-band SSPA \\
\hline HPA IBO [dB] & 0 & 4 \\
\hline HPA OBO [dB] & 0.422 & 1.134 \\
\hline Normalized Carrier Spacing & \multicolumn{2}{|c|}{1.1} \\
\hline Number of Adjacent channels & \multicolumn{2}{|c|}{6} \\
\hline
\end{tabular}

It is worth noting that spectral efficiency computation is based on the carrier spacing, thus the spectral efficiency is defined as $\frac{r \cdot \log _{2}(M)}{B_{c s}}$ where $M$ is the modulation cardinality, $r$ is the code-rate and $B_{c s}$ is the carrier spacing normalized to the symbol-rate.

A first important task to be performed is the optimization of the user SSPA (Solid State Power Amplifier) operating point. In particular, two criteria have been used, which are the compliance with the RCS2 spectral mask [1] and the minimization of the degradation of the useful signal. The first criterion essentially deals with out of band emissions, which are worsened by non linear effects but shall be kept under control, while the second one aims at minimizing the effect of in-band distortion without excessively reducing the transmit power. For the latter task, the total degradation $(T D)$ has been computed, defined as:

$$
T D=\Delta S N R+O B O \quad[d B]
$$

where $\triangle S N R$ is the difference (in $\mathrm{dB}$ ) between the required $S N R$ (signal-to-noise ratio) for a given quality of service in the scenario under investigation and in a linear channel, and $O B O$ is the output back-off of the amplifier, defined as the ratio between output saturation power and output average power. 
Our investigations have shown that QPSK does not suffer from non-linearity issues, respecting the DVB-RCS2 spectral mask without any additional back-off, and yielding a very low degradation even for zero back-off. The best working point in this case is, therefore, the one reported in Table I. On the other hand, in case of 8PSK the emission in the nearest adjacent channels must be limited by significantly backing off the working point in order to respect the spectral mask. Fig. 2 reports an example of TD plot vs. OBO in the absence and in presence of adjacent channel interference (ACI). In the presence of ACI, the best working point is shifted to higher OBO values because the total degradation is affected also by the ACI spectral regrowth as well as by the useful signal distortion. Obviously, the TD does not tend to the OBO straight line (as it does in absence of ACI) because the considered $\mathrm{OBO}$ range is too low for this asymptotic convergence.

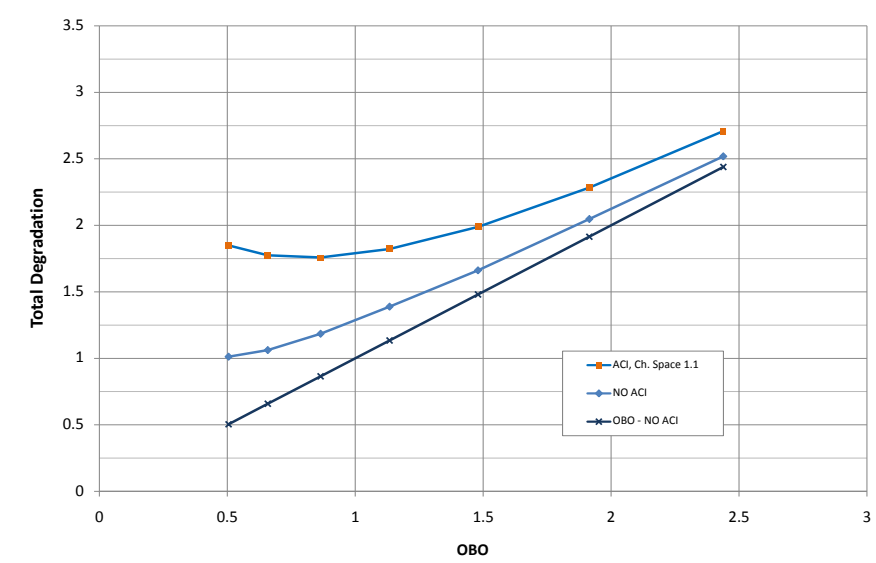

Fig. 2. Total Degradation analysis for LM waveform 8: 8PSK 2/3

Taking into account all such considerations, the LM receiver structure, depicted in Fig. 3, is based on Rife and Boorstyn [9] coarse frequency estimation, with a modification as in [10] to let the estimator operate on different data fields, and on a Gardner timing estimator [11]. The phase noise compensation can be performed by a digital phase locked loop (PLL) [12] operating on both known symbols and hard decisions as in [13], or, when necessary, by the CBC demodulation algorithm [14], which yields finer estimation, being thus advisable for high order modulations, at expense of additional complexity.

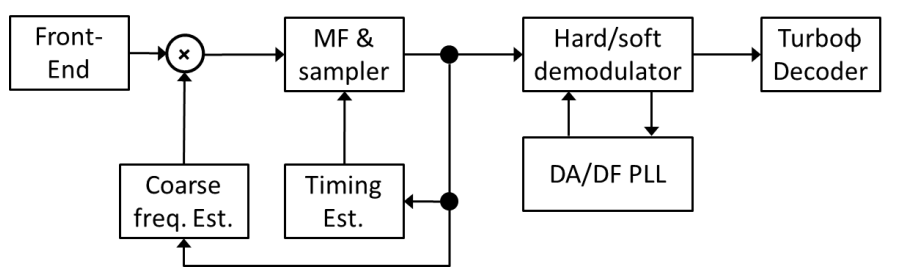

Fig. 3. Block Diagram of the LM receiver

\section{Continuous Phase Modulation Waveforms}

The considered partial response CPM schemes, as per DVBRCS2 [1], are serially concatenated with a convolutional code. Our analysis has started with the analysis of two waveforms which provide spectral efficiencies equal to 0.5 and 1.8 $\mathrm{bit} / \mathrm{s} / \mathrm{Hz}$. A second step in the analysis has led to investigate also waveforms with intermediate spectral efficiencies, as explained in the next section. The waveform parameters for

TABLE II

CPM MODULATION WAVEFORM PARAMETERS

\begin{tabular}{|l|c|c|c|c|}
\hline DVB-RCS2 CPM Waveform ID & 3 & 5 & 7 & 8 \\
\hline Pulse Type & \multicolumn{5}{|c|}{ Q2AV } \\
\hline AV Pulse $\alpha_{R C}$ & 0.98 & 0.75 & 0.75 & 0.625 \\
\hline Modulation index h & $2 / 5$ & $2 / 7$ & $1 / 4$ & $1 / 5$ \\
\hline Code-rate & $1 / 2$ & $2 / 3$ & $4 / 5$ & $6 / 7$ \\
\hline Packet length (bit) & \multicolumn{5}{|c|}{400} \\
\hline Spectral efficiency & 0.5 & 1.1 & 1.5 & 1.8 \\
\hline HPA type & \multicolumn{5}{|c|}{ Ka-band SSPA } \\
\hline HPA IBO [dB] & \multicolumn{5}{|c|}{0} \\
\hline HPA OBO [dB] & 2 & 1.21 & 1.0667 & 0.974 \\
\hline Normalized Carrier Spacing & 2 &
\end{tabular}

all the considered CPM schemes are reported in Table II, in which the pulse type refers to the CPM pulse shape, which is the weighted average (AV) of the raised-cosine (RC) and rectangular (REC) pulse shapes [1], such that

$$
g(t)=\alpha_{R C} g_{R C}(t)+\left(1-\alpha_{R C}\right) g_{R E C}(t)
$$

where both $g_{R C}(t)$ and $g_{R E C}(t)$ have time support $0 \leq t \leq$ $2 T$, and are defined, respectively, as $g_{R C}(t)=\frac{1}{4 T}\left(1-\cos \frac{\pi t}{T}\right)$ and $g_{R E C}(t)=\frac{1}{4 T}$.

The CPM signal can, therefore, be written as:

$$
s(t)=\cos \left[2 \pi f_{c} t+2 \pi h \sum_{k=-\infty}^{\infty} a_{k} \int_{-\infty}^{t} g(\tau-k T) d \tau\right]
$$

where $f_{c}$ is the carrier frequency, $h$ is the CPM modulation index, and $a_{k}$ is the data symbol at time interval $k$.

Note also that because the CPM waveform has constant envelope, the user terminal HPA does not need any input backoff (IBO), hence resulting in no output back-off (OBO).

On the receiver side, in ideal channel conditions, coherent detection based on the Rimoldi decomposition of the CPM signal [15], is performed. When channel impairments are considered, non-coherent detection is instead performed.

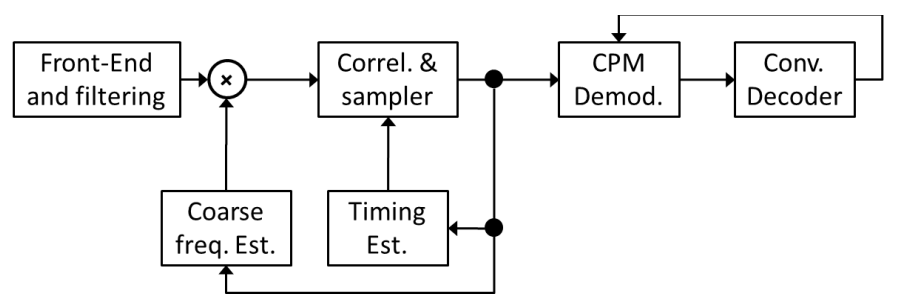

Fig. 4. Block Diagram of the CPM receiver

The CPM receiver for non-coherent detection is reported in Fig. 4. It is based on a phase synchronization technique 


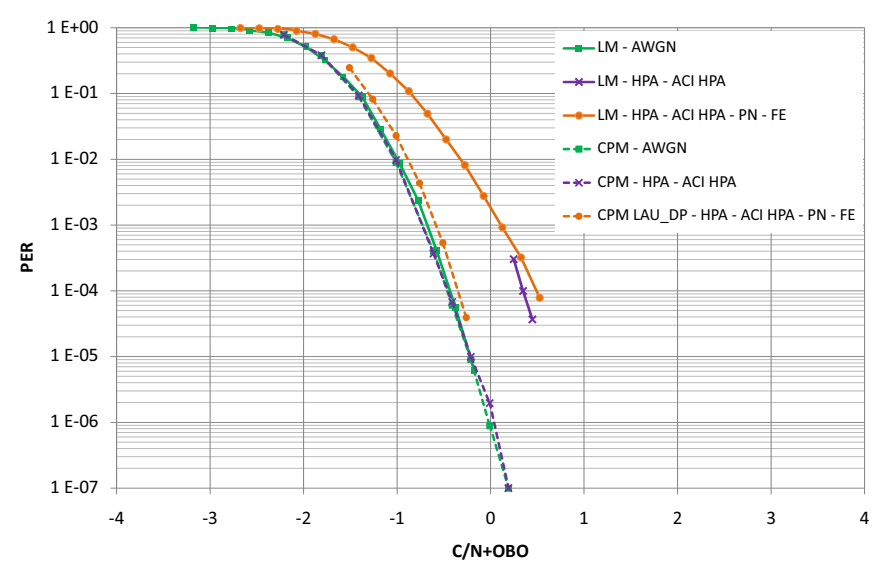

Fig. 5. PER comparison: LM vs. CPM, RCS2 waveform ID = 3, considering phase noise (PN), frequency errors (FE), and ACI

embedded in the BCJR algorithm of the CPM detector leveraging on the Bayesan approach [10], in which a statistical model is assumed for the phase noise process. A Laurent decomposition is used [16], [17] which is truncated to the first $M-1$ components, for complexity reduction, and the phase is discretizated into $R$ values, where $R$ depends on the CPM modulation index $h$. It is worth mentioning that the demodulation process is performed in an iterative fashion together with decoding, yielding a better performance. In addition, the considered CPM non-coherent receiver employs a Rife and Boorstyn coarse frequency estimator [9] and the timing estimator proposed in [18].

\section{Performance AnAlysis}

In this section, numerical simulations of LM and CPM performance are presented and compared.

Fig. 5 and Fig. 6 report performance in terms of PER (Packet Error Rate) as a function of the overall $\mathrm{C} / \mathrm{N}$ (carrier to noise ratio) plus OBO for both CPM and LM, and refer to two spectral efficiencies, 0.5 and $1.8 \mathrm{bit} / \mathrm{s} / \mathrm{Hz}$, respectively.

In Fig. 5, considering the square and cross marked lines (both solid and dashed) it can be seen that CPM performs close to QPSK 1/3 in AWGN. When the effect of the user HPA and of the ACI is considered, CPM outperforms LM by around $1 \mathrm{~dB}$ (at $\mathrm{PER}=10^{-4}$ ). This is due to the fact that CPM waveforms pass undistorted through the user HPAs. This can be appreciated observing the good match between CPM performance with and without HPA and ACI.

The analysis is further extended by taking into account receiver instabilities in the performance assessment. Considering the solid and dashed circle marked lines in Fig. 5, it can be seen that the degradation due to channel impairments is almost negligible, in the order of $0.1 \mathrm{~dB}$ for $\mathrm{CPM}$, bringing the CPM advantage on $\mathrm{LM}$ to $1.1 \mathrm{~dB}$.

In the same channel conditions, CPM and LM are compared for spectral efficiency of $1.8 \mathrm{bit} / \mathrm{s} / \mathrm{Hz}$ in Fig. 6. In this case, CPM shows a significant degradation when ACI is considered

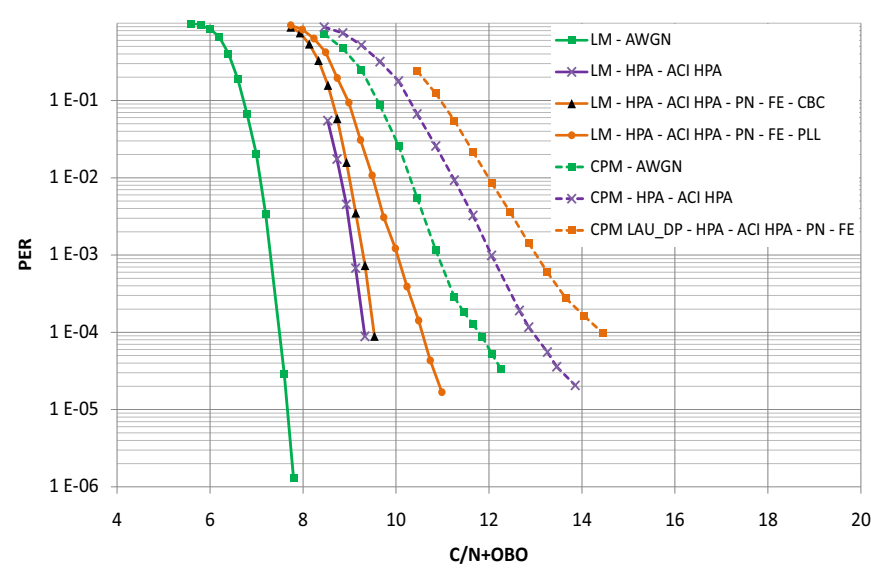

Fig. 6. PER comparison: $\mathrm{LM}$ vs. CPM, RCS2 waveform ID $=8$, considering phase noise $(\mathrm{PN})$, frequency errors $(\mathrm{FE})$, and $\mathrm{ACI}$

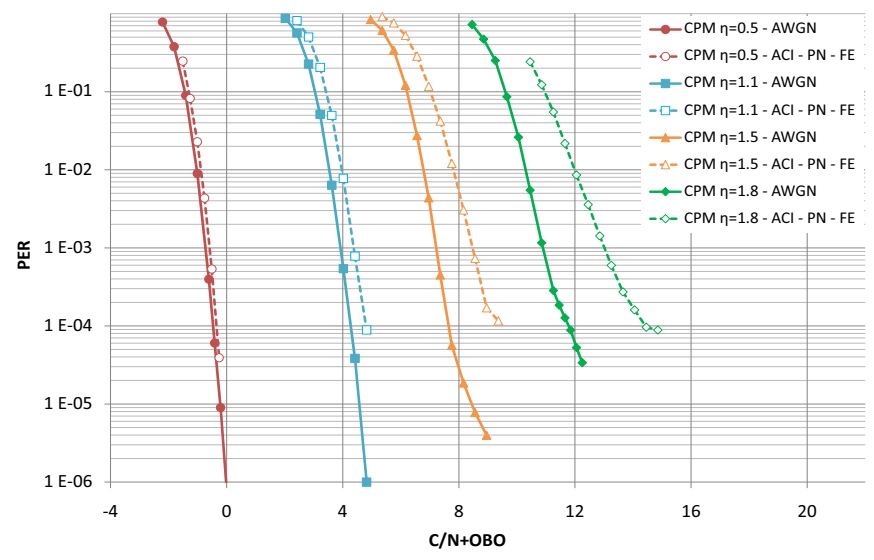

Fig. 7. CPM waveforms comparison

because of the tighter spacing between carrier that is needed to reach such high spectral efficiency. On the other hand, the gap between the ideal AWGN case and the case with ACI and HPA is significantly lower for CPM than for LM, implying that CPM waveform can better cope with user HPA non idealities in the characteristics and in the control of the operating point. Regarding LM, on the other hand, the performance improvement obtained by using a CBC demodulator rather than a PLL is about $1 \mathrm{~dB}$ at $\mathrm{PER}=10^{-4}$, thus confirming the interest in more robust (although more complex) demodulation schemes for high order constellations. When channel impairments are also considered, the same considerations hold true: the overall gap from the AWGN performance is larger for LM than for CPM. This observation suggests to investigate the CPM waveform behavior also for intermediate spectral efficiencies in order to assess performance trends of such promising scheme in the considered channel conditions. Hence, Fig. 7 shows a comparison between CPM waveforms for spectral efficiencies $\eta$ equal to $0.5,1.1,1.5$, and $1.8 \mathrm{bit} / \mathrm{sec} / \mathrm{Hz}$. 


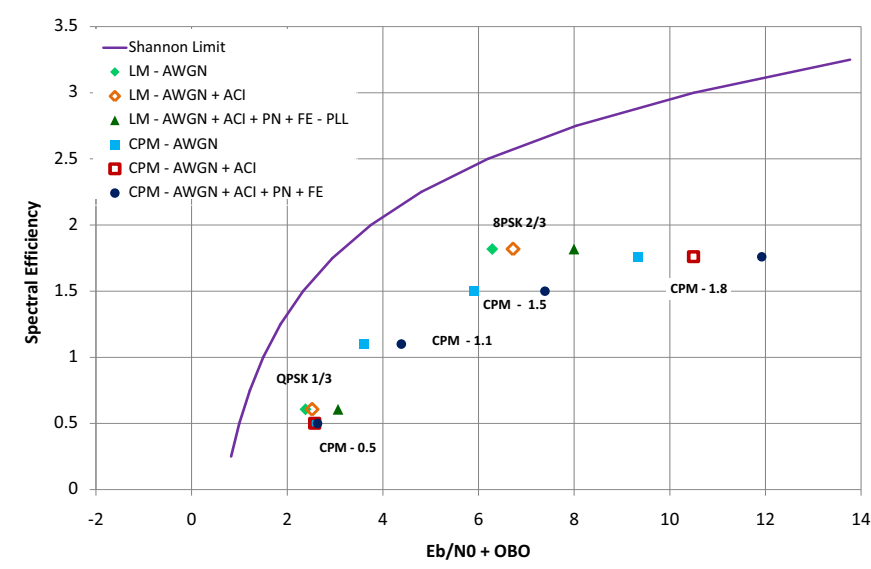

Fig. 8. Spectral Efficiency: CPM vs. LM comparison

As it can be seen, for $\eta=1.1$ the degradation coming from $\mathrm{ACI}$ and non idealities with respect to AWGN is of the order of $0.4 \mathrm{~dB}$, while for $\eta=1.5$ is of around $1 \mathrm{~dB}$. Therefore, both are better resilient to ACI and non idealities than the highest spectral efficiency waveform $(\eta=1.8)$ while still providing significantly better performance in terms of PER. This behaviour can be justified considering that for high SNR the system is ACI-limited rather than AWGN-limited, and that a similar behaviour has been observed in several other studies on CPM modulation, e.g. in [19] .

Finally, Fig. 8 reports all the considered waveforms on the Shannon plane, and it is worthwhile recalling the following ideal conditions assumption used in ths comparison:

- the ACI power is perfectly balanced with the power of the useful signal;

- the ACI HPAs working point is ideally controlled;

- the useful signal HPA working point is the optimal one (i.e., non-ideal SSPA control is neglected).

The above assumptions have a significant impact on the analysis, as they represent an optimistic baseline for the LM performance, hence suggesting that CPM waveforms can be suitable for mesh applications, since such applications are supposed to operate in a low SNR range, and for this case properly designed CPM waveforms do not suffer for any additional degradation due to channel imperfections.

\section{CONCLUSION}

We have presented an analysis of air interfaces performance into a Mesh satellite scenario. Non-linearities, receivers instabilities, channel imperfections (i.e. user terminal characteristics, phase noise and carrier recovery errors) were taken into account in this analysis, along with the effect of adjacent channel interference (ACI) caused by signals directed to other users but in the same multiplex of signals forwarded to the satellite.

We have shown that CPM is potentially suitable for the Mesh scenario under consideration, since it is more resilient to real-life impairments that affect transmissions for low to medium spectral efficiencies. For high efficiency cases, LM waveforms performs better, thanks to higher cardinality and lower coderate (i.e., better protection of data bits). CPM is however more appealing because, thanks to its constant envelope, is prone to mesh mass-market system characterized by low cost devices that will increase the need of robust and resilient waveforms.

\section{ACKNOWLEDGMENT}

This work has been in part supported by the ESA Project $n$. 4000102296/11 "Cost Effective Satellite Terminals for MESH Overlay Networking".

\section{REFERENCES}

[1] DVB BlueBook A155-2 (03/11) DVB-RCS2 Lower Layer Satellite Specification.

[2] T.Ors, Z.Sun, B.Evans, "A Meshed VSAT Satellite Network Architecture using an On-Board ATM switch", IEEE International Performance, Computing, and Communications Conf., 1997, 05-07 Feb. 1997.

[3] "The VSAT Report Comsys 2005", VSAT Industry Status Report to Clients, 2005.

[4] S. Arnold, A. Noerpel, R. Gopal, S. Chandrasekharan, "Implementing a mobility architecture for a Regenerative Satellite Mesh Architecture (RSM-A) system a SPACEWAY perspective", IEEE Military Comm. Conf., MILCOM 2008, 16-19 Nov. 2008.

[5] C. Haardt, "Demonstrating Radio Burst Switching by satellite in the ULISS project", 10th International Workshop on Sig. Proc. for Space Comm., 6-8 Oct. 2008.

[6] O. del Rio Herrero, X. Maufroid, "Innovative Hybrid Optical/Digital Ultra-Fast Packet-Switched Processor for Meshed Satellite Networks", IEEE Journal on Sel. Areas in Comm., Vol 22, pp. 250-260, Feb. 2004

[7] C. Haardt, O. del Rio Herrero, N. Alagha, "Semi-Transparent Packet Switching by Satellite: migrating existing technologies for new opportunities", 26th International Commu. Satellite Systems Conf. (ICSSC), 10-12 June 2008, San Diego, CA.

[8] R. Suffritti, E.A. Candreva, F. Lombardo, S. Rosati, A. Vanelli-Coralli, G.E. Corazza, G. Gallinaro, "A Mesh Network over a Semi-Transparent Satellite", IEEE GLOBECOM 2011, 5-9 Dec. 2011, Houston, TX, USA.

[9] D. Rife, R. Boorstyn, "Single tone parameter estimation from discretetime observations", IEEE Trans. on Inform. Th., vol.20, no.5, pp. 591598, Sep 1974

[10] A. Barbieri, G. Colavolpe, "Simplified Soft-Output Detection of CPM Signals Over Coherent and Phase Noise Channels", IEEE Trans. on Wireless Comm., vol. 6, no. 7, July 2007.

[11] F. Gardner, "A BPSK/QPSK Timing-Error Detector for Sampled Receivers", IEEE Trans. on Comm., vol.34, no.5, pp. 423- 429, May 1986.

[12] Y.R. Shayan, T. Le-Ngoc, "All Digital Phase-Locked Loop: Concept Design and Applications", IEE Proc., vol 136, Pt. F, No. 1, pp 53-56, Feb 1989 .

[13] G. Albertazzi, S. Cioni, G.E. Corazza, M Neri, R. Pedone, P. Salmi, A Vanelli-Coralli, M. Villanti, "On the adaptive DVB-S2 physical layer: design and performance". IEEE Wireless Comm., vol.12, no.6, pp. 6268, Dec. 2005

[14] A. Barbieri, G.Colavolpe, G. Caire, "Joint Iterative Detection and Decoding in the Presence of Phase Noise and Frequency Offset", IEEE Trans. on Comm., vol.55, no.1, pp.171-179, Jan. 2007.

[15] B.E. Rimoldi, "A decomposition approach to CPM", IEEE Trans. on Inf. Th., vol.34, no.2, pp.260-270, Mar 1988.

[16] P. Laurent, "Exact and Approximate Construction of Digital Phase Modulations by Superposition of Amplitude Modulated Pulses (AMP)", IEEE Trans. on Comm., vol.34, no.2, pp. 150- 160, Feb 1986.

[17] U.Mengali, M.Morelli,“Decomposition of M-ary CPM Signals into PAM Waveforms", IEEE Trans. on Inform. Th.,pp 1265-1275,tot.pag 11,1995

[18] A.N. D'Andrea, U. Mengali, M. Morelli, "Symbol timing estimation with CPM modulation", IEEE Trans. on Comm., vol.44, no.10, pp.13621372, Oct 1996

[19] A. Barbieri, D. Fertonani and G. Colavolpe, "Spectrally-efficient continuous phase modulations", IEEE Trans. Wireless Commun., vol. 8, no.3, pp. 1564-1572, March 2009 